\section{Kirchen für Menschen}

\section{Argumente für die Nutzung von reformierten Kirchenbau-} ten $^{1}$

Matthias Krieg

\section{Christliche Kirchen sind wie antike Tempel zwar einer Gottheit gewidmet, wie sie aber für die Benutzung von Men- schen gedacht.}

Der Römer Vitruv hat der Nachwelt etwa eine Generation vor der Geburt von Jesus von Nazareth die einzige Architekturlehre der antiken Welt hinterlassen. In seinen Zehn Büchern über die Architektur ist eines dem Bau von Tempeln gewidmet. Das Verdienst, Vitruvs Vorstellung vom menschgemässen Tempel wiederentdeckt und bis heute nachhaltig bekannt gemacht zu haben, liegt bei Leonardo da Vinci, der 1490 mit seiner Skizze des Vitruvianischen Menschen bildlich sichtbar machte, was Vitruv in Worten hinterlassen hatte: Der statisch stehende Mensch erzeugt das Quadrat, der dynamisch bewegte Mensch den Kreis, und beide Formen göttlicher Vollkommenheit ruhen nach unten, also geerdet, ineinander. Alle Masse und Proportionen des Tempels sollen sich nach Vitruv an diesem Grundmodell orientieren. Noch heute kann man der Skizze begegnen, die eigentlich das Vitruvianische Gotteshaus heissen müsste, denn sie verbildlicht Vitruvs Vorstellung vom Tempel nach menschlichem Mass: der Gottheit geweiht, aber vom Menschen erlebt.

1 Dieser Text entstand am 10. Juli 2018 als Argumentarium für Dekan Pfr. Ernst Hörler in Wädenswil.

Jahrbuch Diakonie Schweiz 3 (2019) - ISSN 2504-3994

Dieser Text ist lizenziert unter einer Creative Commons Namensnennung 4.0 International Lizenz (CC BY 4.0): (https://creativecommons.org/licenses/by/4.0/).
Ohne behaupten zu wollen, dass Jesus, Paulus oder die Evangelisten Vitruvs Schrift gekannt hätten, aber im Wissen, dass das Imperium Romanum um das ganze Mare Nostrum herum eine Kulturgemeinschaft gebildet hatte, auf die Paulus, der älteste schriftliche Theologe, sich ja expressis verbis auch beruft (Apg 21,39), werden neutestamentliche Stellen, die mit Tempelmetaphorik arbeiten, anders lesbar und interpretierbar: Jesu Leib als Tempel (Joh 2,21), der Leib der Christen als Tempel des heiligen Geistes (1Kor 6,19; Offb 3,12), die Gemeinde als Tempel Gottes (1Kor 3,16; 2 Kor 6,16; Eph 2,19-22), Gott selbst als Tempel seiner Gemeinde (Offb 21,22). Bei Vitruv ist der Tempel menschenförmig, im Neuen Testament ist der Mensch tempelförmig.

Der Kirchenbau ist für den Menschen da. Er trägt menschliches Mass, damit sich der Mensch gerne in ibm aufhält. Er soll sein Eigenort sein, seine Idiotopie, mindestens sein Sehnsuchtsort, seine Heterotopie, keinesfalls aber sein Unort, keinesfalls seine Utopie. - Negativ gesagt: Der Mensch ist nicht für den Kirchenbau da!

\section{Architektur und Gestaltung, die als Kunst gelten und} schutzwürdig sind, folgen untrennbar beidem: inhaltlich einem theologischen Konzept und formal funktionalen Anforderungen.

Älteste christliche Kirchbauten, Baptisterien und Grabkirchen, waren Zentralbauten und dienten der Funktionalität des Lebenslaufs, indem sie Geburt und Tod markierten. Mit der Übernahme des Christentums als römischer Staatsreligion kam es zur Übernahme der kaiserlichen Basilika: Das basilikale Schema diente der Huldigung, nun des Pantokrators anstelle des Imperators. In der Romanik wurde die Kirche zum Gleichnis des irdischen Lebenswegs von der Taufe im Westen bis zur Erlösung im Osten: Der Kirchenraum war irdisch, seine Mauern waren schwer, das Licht kam fast nur aus dem Osten, ex oriente lux. Der Raum barg und führte. In der Gotik wurde die Kirche zum Gleichnis des Himmels: Lastende Wände wurden leicht und transparent, der Blick wurde in die Höhe gezogen, und 
die Decke war ein luftig flatterndes Zelt. Klosterkirchen gehörten stets zu einem Ensemble von Bauten klösterlichen Lebens: In ihnen waren Funktionen am wenigsten ablösbar von Architektur und Gestaltung.

Was hier nur angedeutet bleibt, ist für die Nutzung des Kirchenraums entscheidend: Jede Epoche entwickelte kontextuell ein theologisches Raumkonzept, das gleichzeitig Funktionalität gewährleistete. Der Kirchenbau war so etwas wie eine Gesamtmetapher und zugleich stellte er ganz praktische Nutzbarkeiten sicher. Er diente der Lebensgemeinschaft und zeigte ihre Vision. - Allerdings ging zuerst der Kirche selbst dieses Wissen über Sinn und Geist ihrer Bauten verloren. Die Epoche der Neostile 1850-1920 zeugte für diese Verlegenheit und diesen Verlust. Inzwischen hat sich zwar die Kirche weitgehend vom Historismus befreit, nicht aber die Denkmalpflege.

Aufgabe der Kirche ist es, Konzept und Funktion mit Leben zu erfüllen, Aufgabe der Denkmalpflege, solches Leben zu ermöglichen und mit der Schutzwürdigkeit in Einklang zu bringen. - Negativ gesagt: Eine Kirche ist keine l'art pour l'art, kein artifizieller Selbstžpeck, kein historisches Museum.

\section{Das reformierte Kirchenbauverständnis beruht, wie be- reits der Bernhardische Plan, auf der Ästhetik von Reduk-} tion und Differenz.

Bernhard von Clairvaux hatte sich im Jahre 1125 in seiner Apologia ad Guillelmum scharf gegen die Bilderflut und Ausstattungswut der Cluniazenser ausgesprochen, damit auch gegen den monastischen Wettbewerb mit Papst und Kaiser um die Entfaltung von Pracht und Macht zu hegemonialen Zwecken. Vierhundert Jahre vor der geordneten Demontage der Bilder und Devotionalien in Zürcher Kirchen hat Bernhard den Kirchenbau von politischem Missbrauch schützen und ihn der Lebensgemeinschaft des Ordens zurückgeben wollen. Das Leben der Gemeinschaft stand in deutlicher Differenz zur Vorherrschaft Einzelner. Bernhard verbot alle basilikalen Motive und jede magische Bildverwendung. Er entwarf ein architektonisches Grundkonzept des Klosterensembles, den Bermbardischen Plan, dem europaweit alle Filialen der vier Mutterabteien zu folgen hatten. Zuwiderhandelnde Äbte wurden bestraft. Bernhards Grundgedanke war die Reduktion, der eremitische Rückzug in die Wüste: Er wollte das Material sprechen lassen, den Stein, das Wasser, den Ton, das Glas. Kunst entstand auch hier aus klarer Funktionalität, nun aber auch aus reduzierter Ästhetik. Reduktion statt Redundanz, Differenz statt Kompetition. Während sich die Zisterzienser in die Eréme zurückzogen, um an einem kleinen Bach aus Osten und versteckt in unspektakulärer Landschaft neu zu bauen, entrümpelten die Schweizer Reformatoren bestehende Kirchbauten vom religiösen Brimborium der Jahrhunderte. Die Schweizer Reformation war theologisch und ästhetisch eine gigantische Ausnüchterung zugunsten des Wesentlichen, das in den fünf Soli zum Ausdruck kam: sola scriptura, gratia und fide, solus Christus und soli Deo gloria. In reformierten Neubauten wie St. Peter in Zürich sind die soli am sichtbarsten.

Fünfhundert Jahre nach dem Beschluss des Rats von Zürich ist eine erneute Rückgabe der Kirchbauten an die Lebensgemeinschaft angezeigt. Heute sind nicht Redundanz und Magie das Problem, sondern Erstarrung und Fixierung: Architektonisch und gestalterisch entwickelte sich seit dem Ende der Neostile zwar Neues, aber ohne genügende Partizipation der Lebensgemeinschaft, nicht selten sogar gegen sie. Deshalb werden nach ihrer Profanierung oder Umwidmung nicht selten solche Kirchen abgerissen, die im Zeitraum 1930-70 gebaut wurden. Die Kirchgemeinden waren nicht beteiligt und hatten sie nie angenommen. Sie sind noch immer auf die Epochen vor 1850 fixiert. Kirchliche Kultur ist zur Zeit der Neostile erstarrt und hat sich nicht lebendig weiterentwickelt. Dafür sprechen auch die Fixierung der Gemeinden auf die Orgel als das eine Kircheninstrument und die Fixierung der Denkmalpflege auf die Bänke, die der griechischrömischen Kampfordnung der Infanterie entsprechen, der Phalanx. War die Kirche, der Bernhard durch Reduktion und Differenz widersprochen hatte, die ecclesia triumphans seiner Zeit, so ist die Kirche, der heute durch Reduktion und Differenz zu widersprechen wäre, die ecclesia militans, die 
zur Zeit der Staatsreformen, etwa um 1850 also, anachronistisch erstarrt ist.

Der Kirchenbau dient dem Leben der gegenwärtigen Gemeinde. Wenn er hingegen weit deutlicher vergangenes Leben einer vergangenen Zeit repräsentiert, dann ist er nach den Kriterien von Reduktion und Differenz aus seiner anachronistischen Erstarrung zu befreien und ins gegenwärtige Leben zu überführen. - Negativ gesagt: Die Kirche ist nicht die Handlangerin der Denkmalpflege.

4. Reformierte Kirchenräume sind weder konsekriert und sakral durch das magische Ritual der Weihe noch profaniert und profan durch das magische Ritual der Entweihung, sondern sie werden immer erst heilig, nämlich theologisch durch die unverfügbare Gegenwart des Heiligen Geistes.

Das Urmodell der Heiligkeit, die sich aus der souveränen Gegenwart der göttlichen Kraft ergibt, ist in der Berufungsgeschichte des Mose überliefert (Ex 3,1-5): Gott erscheint im Feuer und entzündet einen Dornbusch. Dies ereignet sich gerade nicht an einem für Gott vorgesehenen Ort oder in einem für Gott gebauten Tempel, sondern im gewöhnlichen Nomadenalltag der Steppe. Die Heiligkeit des Ortes ist ein momentum, ist die heilige $W$ ille, die wieder vergeht. Heiligkeit ist das göttliche Sichereignen, es kann nicht angeeignet und festgeschrieben werden. Gott ist souverän und nich domestizierbar. - Für den reformierten Gottesdienst bedeutet dies, dass nur dann, wenn Gottes Wort einen Menschen erreicht, ihn anspricht und in eine existenzielle Wende bringt, die Zeit und der Raum, in denen dies geschieht, heilig sind. Wenn einer das Wort hört und versteht, ist dies seine beilige Weile. Das muss nicht in einer Kirche passieren und kann auch an jedem anderen Ort passieren. Deshalb kann die vom Zwang zur Heiligkeit befreite Kirche multifunktional genutzt werden, sonntags wie alltags, wie der Steppenboden der Sinai oder der Brunnen bei Sychar (Joh 4).

Theologisch entscheidend für einen lieu d'église ist die Möglichkeit der Begegnung mit dem Heiligen. Kirchen sind Orte potenzieller Begegnung.
Die Kirchgemeinde trifft sich für dieses Potenzial und wegen der Verheissung dieses Potenzials. Reformierte Liturgie ist nicht mehr magisches Abbild eines religiösen Urbilds wie in der Orthodoxie oder magische Wiederholung des Opfers am Kreuz wie in der römischen Liturgie, sondern theologische und ästhetische Wegbereitung für das Kommen des Heiligen Geistes und die Begegnung mit ihm. Architektur und Gestaltung, Liturgie und Predigt sind in der reformierten Tradition Darstellung von Potenzialität. Mit dem Bau von Kirchgemeindezentren den Ort der Begegnung und die Potenzialität der Begegnung aus historischen Kirchgebäuden weitgehend ausgelagert und somit zu deren spiritueller Verödung erheblich beigetragen zu haben, ist ein ekklesiologischer Sündenfall der Kirche seit 1950. Das christliche Grundgebot der Gastfreundschaft gilt bereits für das Kirchgebäude und nicht erst für das Kirchgemeindehaus!

Der reformierte Kirchenraum ist diesseits der vorreformatorischen Unterscheidung von sakral und profan, drinnen und draussen, intra muros und fiori le mure multipel nutzbar, nämlich als Ort potenzieller Begegnungen. - Der Kirchenraum ist nicht magisch aufgeladen und daher auch nicht unantastbar!

\section{Die multifunktionale Nutzung von Kirchenräumen hat sich in der Reformation ergeben, nämlich ekklesiologisch aus der Aufwertung der Gemeinde zum Subjekt ihrer Kirche und soziologisch aus der Alleinstellung des Kirchgebäudes am Ort.}

Die reformierte Reformation war eine urbane Bürgerbewegung. Sie nutzte das bestehende Selbstbewusstsein freier Reichsstädte. Die Bürger verlangten wie schon 1414 in Prag nun auch 1523 in Zürich ihr Selbstbestimmungsrecht auch in Fragen der Religion. Die öffentliche Disputation war das Kommunikationsinstrument in reformierten Regionen. So kam es zu einer über viele Generationen nahezu vollständigen Identität von kirchlicher Christengemeinde und politischer Bürgergemeinde. 1850 waren 97.7\% der Zürcher Bevölkerung reformiert, 2016 waren es nur noch
Jahrbuch Diakonie Schweiz 3 (2019) - ISSN 2504-3994

CC by 4.0 
29.6\%. Nicht nur die Klärung aller Belange der Polis fand im einzigen öffentlichen Raum der Kommune statt, er war ihr auch Festsaal, Tanzboden, Marktplatz, ihre Gerichtsstätte, Notunterkunft, Fluchtburg etc. Nachdem die Reformation dem Kirchenraum die magisch verstandene Sakralität genommen hatte, gab sie ihn der Gemeinschaft der Gläubigen als Begegnungsort zurück. Der politischen Profanierung der Kirche als eines Ortes, an dem sich die Hierarchie darstellte, folgte ihre ekklesiologische Kommunisierung als eines Ortes, der einer Lebensgemeinschaft gehört und von ihr belebt wird.

Gemeinde Jesu Christi ist die Kommunikationsgemeinschaft der Glaubenden. Communio entsteht aus communicatio. Kommuniziert werden das Wort und das Mahl. Beide waren seit 1531 und 1414 kommunisiert. Der Priesterstand hatte seine beiden Grundprivilegien, den exklusiven Zugang zum Wort und zum Mahl, verloren. Entsprechend ist der reformierte Kirchenbau nicht mehr der Ort von Proskynese und Messopfer, sondern der Lebensort der Gemeinde. Nicht mehr die repetitio des Selbigen prägt den Ort, sondern die variatio des Gleichen. Damit wird er allerdings gerade nicht zum privaten Wohnzimmer oder Vereinslokal zweier dominanter Lebenswelten von zehn, wie dies heute länger schon der Fall ist, sondern innerhalb einer Mixed Economy of Church, die allen zehn Lebenswelten Begegnung ermöglicht, wird er jeweils zum öffentlichen Ort einer Lebensgemeinschaft, deren Gestaltung ein öffentliches Bekenntnis ist.

Der reformierte Kirchenraum ist Begegnungsraum der Kirchgemeinde. Sie ist sein Subjekt, und Haushalterschaft im Interesse des Gemeindelebens ist ibre Aufgabe. Gemeindeleben unter den Bedingungen der Postmoderne geschieht in einer Mixed Economy of Church. In ibr werden Kirchenorte im Rahmen lebensweltlicher Expressivität als Begegnungsräume von Lebenswelten gestaltet. - Negativ gesagt: Landeskirche und Denkmalpflege, die Lebenswelten die Begegnung in ibren Eigenorten versagen, haben ibren Auftrag verfeblt und daher versagt.
6. Kirchenräume haben wie Tempel eigentlich keine Sitzgelegenheiten, denn der heilig gewordene Raum des Vor-GottSeins ist, religionspsychologisch betrachtet, der Ort der Proskynese, der Unterwerfung, weshalb der Mensch vor der Gottheit steht oder kniet, während sie es ist, die sitzt.

Gemäss platonischer Ständelehre gab es für den Dritten Stand keine Kirchenbänke. Das Volk stand, wie heute noch in Kirchen der Orthodoxie. Nur für Alte und Kranke gab es allenfalls transportable Bänke. Der Klerus als Erster Stand hatte im Fall der Bischofskirche und der Klosterkirche sein Chorgestühl. Es stand im Chor, der durch Chorschranken vom Volk getrennt war. Der Adel als Zweiter Stand verfügte über seine erhöhte Loge, wenn nicht gleich über seine eigene Kapelle. Erst mit der Französischen Revolution von 1789 zerfiel die Dreiständelehre. Um 1700 setzte der Einbau fixer Kirchenbänke ein.

Reformierte Kirchen führten die Bank fürs Presbyterium ein. Der Temple de Charenton, 1623 vor den Toren von Paris als Urform der reformierten Kirche erbaut, war bereits keine Basilika mehr, also kein Raum des Huldigens vor dem römischen Kaiser bzw. dem Pantokrator, sondern ein gigantisches Auditorium für 36000 Leute, also ein Raum des Hörens auf das Wort, das von der Kanzel, die nun anstelle des Altars in der Mitte thronte, nach allen Seiten erging. Reformierte Kirchen in den Niederlanden, deren Architektur vorreformatorisch war, waren unmöbliert, wie Malerei des 17. Jahrhunderts zeigt. Die Gemälde machen die Stadt in der Stadt sichtbar, die civitas Dei in der civitas terrena: Menschen bewegen sich in der Kirche, als flanierten sie über den Platz einer Stadt. Sogar Hunde streunen über ihn hin. Der Kirchenraum wird erlebbar als urban und multipel bevölkerbarer Platz oder als beliebig und flexibel gestaltbare Bühne. Nichts ist fixiert. Für Szenen des Zwinglifilms 2019 wurden im Mai 2018 die Bänke im Zürcher Grossmünster daher eigens ausgebaut.

Der offene Kirchenraum kennzeichnet die reformierte Auffassung der kirchlichen Arcbitektur. Räume sind offen für Begegnungen, sind multipel und variabel, belebbar 
und bespielbar, sind Vorgeschmack der civitas Dei mitten in der civitas terrena, sind Ausdruck der Lebendigkeit von Gemeinde. - Negativ gesagt: Je geschützter und gepflegter der Kirchenraum, desto letaler das Gemeindeleben.

7. Im 19. Jahrhundert kommt es in dem Masse zu einer reaktionären Entfunktionalisierung des Kirchenraums, wie es zu seiner exklusiven Nutzung kommt: entweder rekatholisierend als Oratorium oder reorthodoxierend als Auditorium.

Die historisch erste Säkularisierung setzte 1555 mit dem Augsburger Religionsfrieden und der Formel cuius regio eius religio ein: Sie führte zur Territorialisierung der Religion und zur Konfessionalisierung des Staates (Casanova). Die liturgische Hoheit lag beim Fürsten, der das Bekenntnis seiner Untertanen festlegte. Im Gegenzug erhielten Untertanen das Recht, das Territorium zu wechseln und sich in einem solchen niederzulassen, das ihr Bekenntnis hatte. Der weltweite Réfuge der Hugenotten fusste darauf. - Die historisch zweite Säkularisierung begann 1803 mit dem Reichsdeputationshauptschluss, dem letzten Gesetz des Heiligen Römischen Reichs Deutscher Nation, das 1806 unterging: Es enteignete freie Städte, Klöster und Staatskirchen, um deren Ländereien solchen Fürsten zu übergeben, die im Verlauf der napoleonischen Wirren ihren Grundbesitz verloren hatten.

So blieb bis heute das Territorialprinzip seit dem Frieden von 1555 zwar erhalten, aber die wirtschaftliche Basis der Kirchen verlagerte sich seit dem Gesetz von 1803 substitutiv auf Steuern und Rechtstitel. Gleichzeitig mit diesem politisch bedingten wirtschaftlichen Niedergang der Kirchen kam es in ihnen zu reaktionären Rückbesinnungen. Sie schränkten die Multifunktionalität des Kirchenraums zunehmend ein zugunsten einer Rückbesinnung auf das klassisch-katholische Oratorium, in dem nur gebetet, oder auf das orthodox-reformierte Auditorium, in dem nur gehört wird. Kirchen wurden wieder exklusiv zu Stätten des Gebets oder der Predigt. Sie gewannen ganz gegen die Reformation den Anschein der Heiligkeit und Unantastbarkeit zurück.
Die Kirche im Dorf muss wieder zur Kirche für das Dorf werden. Das Gemeindeleben unter den Bedingungen der Gegenwart muss die Nutzung des historischen Kirchenraums bestimmen. - Negativ gesagt: Anachronistische Nutzung von Kirchgebäuden ist per definitionem zukunftslos.

\section{Nach reformiertem Verständnis von Zeit und Ewigkeit} gibt es in der Geschichte zu keinem Zeitpunkt einen normativen Idealzustand, der nicht mehr zu übertreffen wäre.

Reformierte verstehen Zukunft eschatologisch. Die Vollendung des Lebens liegt vorn. Das Beste kommt erst noch. Es kann sich zwar in der Zeit ankündigen, als ein Angeld wie bei Paulus, eine Anzahlung aufs Ganze, in seiner Fülle aber ist es für nach der Zeit verheissen, bleibt damit Teil des Glaubens und wird keinem Schauen zugänglich. Zu allen Zeiten hatte christlicher Kirchenbau den Verweischarakter von Architektur und Gestaltung gewahrt: Der fehlende römische Kaiser in der Apsis der Basilika verwies auf den erhöhten Christus als den eigentlichen Pantokrator. Die Kunst der Kirche ist semantisch, nicht deskriptiv. Wenn aber jede Zeit in ihrem Kontext und mit ihren Mitteln nach vorn verweist, dann gibt es keinen Zeitpunkt, der vollendet, unüberbietbar und abgeschlossen wäre, keinen Idealzustand, der idealer nicht mehr werden könnte. Daher gibt es auch keinen Schnitt in der Geschichte, der abschliessend normativ wäre und auf den alles Künftige sich zurückbeziehen müsste. Auch für Reformierte gibt es Referenzen, doch immer nur relative und nie absolute.

Was theologisch so ist, hat rechtliche Folgen: Keine Architektur, keine Gestaltung, kein Utensil in der Kirche kann Anspruch auf Ewigkeit erheben, auf Unverbesserlichkeit und Unveränderlichkeit. Auch obne Orgel ist eine Kirche eine Kirche, auch mit installierter Popelektronik ist sie eine Kirche. Auch obne Bilder und Bibelverse ist sie eine Kirche, aber auch mit Graffiti und einer Halfpipe ist sie eine Kirche. Historische Kirchenräume tragen zu Recht die Spuren der Geschichte, die von vergangenem Leben und vergangener Funktionalität zeugen. Es kann nicht Aufgabe der Denkmalpflege sein, einen historischen Zeitpunkt für normativ zu erklären und 
ein Kirchgebäude historistisch an ihm einzufrieren. Es ist aber sehr wohl die Aufgabe der Denkmalpflege, und zugleich ist es ihr professionelles Können, schützenswerte Spuren der Geschichte sichtbar zu machen, ohne damit zeitgenössisches Leben zu verhindern. Der beste Schutz eines Baus ist das Leben, das in ihm gelebt wird. Unbelebte Räume sind gefährdet und kosten wesentlich mehr als belebte.

Im fünfhundertsten Jahr nach der Reformation besteht die reformierte Kirche darauf, eine ecclesia reformata semper reformanda zu sein. Das schliesst die Fixierung auf ideologische und ästhetische Idealmomente aus und öffnet die Räume für zeitgenössische Gestaltungen des Gemeindelebens in Kirchgebäuden. - Negativ gesagt: Eine historistisch orientierte Denkmalpflege vertreibt Leben aus Räumen und ist daher politisch anzufechten.

\section{Wie das denkmalgeschützte Wohnhaus trotz seiner} Schutzwürdigkeit Privatheit und Familienleben ermöglicht, so muss auch die denkmalgeschützte Kirche trotz ihrer Schutzwürdigkeit Öffentlichkeit und Gemeindeleben ermöglichen.

Schutzwürdigkeit und Belebtheit schliessen sich nicht aus. Das zeigen denkmalgeschützte Privathäuser. Wenn ein Haus aus dem Jahr 1827 inzwischen Strom und Wasser hat, eine Zentralheizung und sanitäre Anlagen, so mussten auch diese Zeugen modernisierten Lebens harmonisiert werden mit den Geboten der Schutzwürdigkeit. Die Denkmalpflege muss die Privatheit des Hauses respektieren und die Entwicklung des Familienlebens ermöglichen. Dasselbe gilt für öffentliche Räume wie Rathäuser, Palazzi oder Museen. Auch in ihnen gibt es Kompromisse, aktuell etwa im Blick auf die Nutzbarkeit durch Rollstühle. Auch in ihnen sind Lebendigkeiten und Belebtheiten zu respektieren.

Die Landeskirche beteiligt sich daher intitiativ und aktiv an der Erstellung eines Katasters über alle Kirchgebäude im Kanton Zürich. Sie redet mit und vertritt gegenüber staatlichen Organen die Interessen ihrer Kirchgemeinden, und zwar im Blick auf ein potenzielles, also zeitgenössisches und zukunftsfähiges Gemeindeleben. Dafür steht der Begriff der Mixed Economy of Church, in der das vorhandene Potenzial an Kirchgebäuden lebensweltlich und konzeptionell als ekelesiologisches Potenzial in den Blick zu nehmen ist. Kirchliche Organe treten also weniger als ökonomisch und konservativ orientierte Liegenschaftenverwaltungen auf, sondern mehr als haushälterische und innovative Investoren in künftiges Gemeindeleben. Kirchgebäude sind nicht nur traditionales Erbe, das zu bewahren ist, sondern auch missionales Potenzial, in das zu investieren ist! Um sich einen Überblick zu verschaffen, wie Denkmalpflegen in benachbarten Kantonen und Ländern mit schutzwürdigen Anlagen, darunter Kirchgebäuden, verfahren, vor allem, um auch andere als historistisch eingeschränkte Vorgehensweisen kennenzulernen, besuchen Organe der Landeskirche Beispiele und Verantwortliche vor Ort und erweitern so ihr Argumentarium.

Die Landeskirche versteht ihre beiden grossen Ressourcen, Kirchenräume und Arbeitszeiten, nicht mehr traditional im Sinn der Bewabrung des Überkommenen, sondern missional im Sinn der Ermöglichung von Kommendem. Darin ist die Reformata auch die Reformanda. - Negativ gesagt: Eine Kirche obne Mission endet.

\section{In einer zeitgemässen Stadtentwicklung sind Denkmal- pflege und Kirchenpflege partnerschaftliche Stakeholder ei- nes grossangelegten politischen und daher öffentlichen Ent- wicklungsprozesses im Interesse der Gesamtbevölkerung.}

Stadtentwicklung und Kirchenentwicklung unterliegen denselben Bedingungen der Postmoderne. Sie haben sich mit vergangenen und gegenwärtigen Narrativen auseinanderzusetzen, um künftige Narrative zu ermöglichen. Sie müssen schützenswerte Monumente und Spuren der Geschichte bewahren, um Platz zu schaffen für neue. Sie müssen die Soziologie und Psychologie ihres Kontextes ergründen, um von Anbeginn an Partizipation im Sinn einer starken Demokratie (Barber) zu gewähren. Nur so lässt
Jahrbuch Diakonie Schweiz 3 (2019) - ISSN 2504-3994

CC by 4.0 
sich die dialektische Aufgabe erfüllen, erstens Wertvolles zu bewahren, zweitens Künftiges zu erkennen und deshalb drittens heute Möglichkeiten zu eröffnen.

Damit es nicht dabei bleibt, dass jede einzelne Kirchenpflege von der kantonalen Denkmalpflege historistisch und im Stil des divide et impera in den Senkel gestellt wird, bedarf es kantonal von Behörde zu Behörde, also zwischen Kirchenrat und Denkmalpflege, eines Rahmenabkommens oder Mantelvertrags. Er folgt dem Grundsatz, dass in einem Bau trotz seiner Schutzwürdigkeit Leben nicht nur negativ zugestanden, sondern auch positiv ermöglicht wird. Dafür wird die Beweislast umgekehrt: Die Denkmalpflege erstellt für jeden Bau wie jetzt schon das Inventar, muss neu aber nachweisen, erstens, wieso etwas schutzwürdig ist, und zweitens, wieso im Negativfall eine ermöglichende Lösung nicht denkbar ist. Da die Ausstattung mit Bänken im Blick auf die Geschichte des Tempel- und Kirchenbaus sehr jungen Datums ist und die meisten reformierten Kirchen Übernahmen aus vorreformatorischer Zeit sind, wird in der Regel keine spätere Möblierung mehr geschützt.

Die Kirchenleitung nimmt ibre politische Aufgabe wabr und erwirkt eine politische Lösung, die das Potenzial der Kirchenräume flexibel nutz̧bar macht. - Negativ gesagt: Lebens- und zukunftsorientierte Nutzungen von denkmalgeschützten Kirchenräumen für das Gemeindeleben können nicht subsidiär ermöglicbt und daher nicht von oben nach unten delegiert werden.

Autor.

Matthias Krieg, Pfr. Dr. phil., Dr. theol, Sekretär der Reformierten Kirche Zürich 